\title{
Análisis de las técnicas neuronales utilizadas en aislantes de transformadores
}

\author{
Josué Quiroga \\ ORCID: https://orcid.org/0000-0001- \\ $6819-56743$ \\ josueesteban_12@hotmail.com \\ Universidad Politécnica Salesiana, \\ Facultad de Ingeniería Electrónica \\ Quito-Ecuador
}

\author{
Juan Joel Segura D' Rouvel \\ ORCID: https://orcid.org/0000-0002- \\ 8763-5513 \\ juan.segura@uti.edu.ec \\ Universidad Tecnológica Indoamérica \\ Quito-Ecuador
}

Recibido (09/11/20), Aceptado (18/11/20)

\begin{abstract}
Resumen: Este artículo presenta el Análisis de Descargas Parciales empleando Técnicas Neuronales. Las máquinas rotativas empleadas en la industria suelen presentar fallas en los aislamientos causadas por falta de mantenimiento y desconocimiento del estado de los mismos. Es importante la realización de pruebas periódicas y evaluaciones continuas del estado del aislamiento para garantizar el correcto funcionamiento de las máquinas. Uno de los métodos empleados para la detección de estas fallas es el de Descargas Parciales. Las cuales consisten en pequeñas descargas producidas en una porción de gas que queda disuelto en el aceite o dieléctrico que constituye el aislamiento de las máquinas eléctricas. En este trabajo de investigación se realiza un análisis de dos trabajos desarrollados en torno a las descargas parciales, donde se han implementado técnicas de inteligencia artificial. Los resultados mostraron una alta efectividad de las redes neuronales para lograr la clasificación de las descargas parciales y aportar en el mantenimiento de equipos eléctricos de alta potencia.
\end{abstract}

Palabra Claves: Equipos eléctricos de alta tensión, descargas parciales, redes neuronales, fallas.

\section{Neural techniques for transformer insulator analysis}

\begin{abstract}
This article presents the Analysis of Partial Discharges using Neural Techniques. Rotating machines used in industry tend to have insulation failures caused by lack of maintenance and ignorance of their status. It is important to carry out periodic tests and continuous evaluations of the state of the insulation to guarantee the correct operation of the machines. One of the methods used to detect these faults is Partial Discharge. Which consist of small discharges produced in a portion of gas that is dissolved in the oil or dielectric that constitutes the insulation of electrical machines. In this research work, an analysis of two works developed around partial discharges is carried out, where artificial intelligence techniques have been implemented. The results showed a high effectiveness of neural networks to achieve the classification of partial discharges and contribute to the maintenance of high-power electrical equipment.
\end{abstract}

Keywords: High voltage electrical equipment, partial discharge, neural networks, failures 


\section{I.INTRODUCCIÓN}

En ingeniería eléctrica una descarga parcial es una ruptura de la rigidez dieléctrica muy localizada del aislamiento líquido o sólido. A diferencia del efecto corona, que se manifiesta en los conductores de una forma más o menos estable, las descargas parciales tienen una naturaleza mucho más esporádica. Las descargas parciales en un material aislante suelen iniciarse en huecos rellenos de gas dentro del dieléctrico. Una vez dieron comienzo las descargas parciales se produce un deterioro progresivo de los materiales aislantes, pudiendo causar a la postre el fallo del aislamiento.

Las descargas parciales en máquinas de gran potencia pueden presentarse de cuatro tipos fundamentalmente; descargas de ranura, descargas superficiales, descargas barra a barra y descargas internas. Cada una de las cuales presenta características propias que la distinguen de las otras. También es posible encontrar en una máquina dos fallas simultáneamente, presentando de esta manera un patrón mixto.

Algunos autores [6] afirman que la mayoría de las empresas utiliza operadores humanos para la clasificación de los patrones de descargas parciales. Este tipo de operadores podría arrojar notorios errores en la clasificación, sobre todo si se trata de personal sin experiencia. Esto conduce a la necesidad de buscar operadores automatizados que ofrezcan mejores prestaciones a la hora de clasificar las fallas de los transformadores y poder realizar las labores de mantenimiento respectivo.

En este artículo se presenta el análisis de las publicaciones presentadas por Suárez [6], [7] donde se desarrolla el diseño de un sistema basado en redes neuronales que permita el análisis de las fallas de los transformadores, a partir de las descargas parciales que éstos arrojan. Este sistema utiliza la herramienta MATLAB para el procesamiento de datos.

El toolbox de redes neuronales artificiales de Matlab ofrece una amplia versatilidad para la implementación de este tipo de sistemas. Además es una herramienta que permite realizar varios tipos de redes neuronales para determinar el menor error del sistema, ofreciendo una convergencia con los valores establecidos.

Los autores [6\}, \{7] presentan dos técnicas para clasificar y analizar fallas en transformadores de potencia; una de las técnicas ofrece un análisis a partir de las matrices de pesos de los patrones de descargas parciales mientras que la otra técnica realiza el proceso a través del análisis de la imagen en particular. Finalmente, se muestran ambos resultados como posibles clasificadores y se evalúa el porcentaje de error para cada caso.

\section{II.DESARROLLO}

Durante el proceso de fabricación de un aislante, pudiera producirse que quedaren pequeñas cantidades de gas aprisionadas en el seno del material, formando cavidades de formas y dimensiones muy diferentes.

Por otra parte, al montar un aislante alrededor de piezas conductoras en equipos eléctricos; por descuido, imperfecciones, dificultades constructivas, pudieran quedar cavidades entre el dieléctrico sólido y los conductores o entre diferentes capas de aislamiento sólido.

El envejecimiento del material aislante ya sea porque frente a solicitaciones externas de los conductores éstos ejercen fuerzas mecánicas o incluso bajo la acción de contracciones térmicas tales, que dan origen a cavidades de tamaño y formas diversas. Los fenómenos de origen eléctrico que se producen en estas cavidades (burbujas o espacios con gas) son fundamentalmente, los mismos.

Cuando el material aislante es sometido a una diferencia de potencial y debido a la presencia de un campo eléctrico el cual tiende a concentrarse en la cavidad, se produce la ionización del gas presente en éstas, dando origen a una descarga eléctrica, conocida como descarga parcial.

Muchas de las salidas de servicio de máquinas rotativas son causadas por falta de mantenimiento y desconocimiento del estado del aislamiento. De ahí la importancia de la realización de ensayos periódicos y las reparaciones necesarias.

El estudio de las redes neuronales artificiales se engloba dentro de la rama subsimbólica de la inteligencia artificial, dicha rama se dedica al estudio y desarrollo de simulaciones de los elementos inteligentes de la naturaleza (cerebros) con el fin de que de forma espontánea estas simulaciones se comporten de forma inteligente.

Así pues partiendo del estudio biológico del cerebro humano o animal las redes neuronales artificiales intentan simular su estructura y funcionamiento. Si en un cerebro el elemento básico es la neurona, en una red neuronal artificial el elemento básico es la neurona artificial, se debe conocer el funcionamiento de la neurona natural para simularlo en la neurona artificial. 


\section{A.Antecedentes}

Desde hace más de 30 años se han estado usando los sistemas de monitoreo de descargas parciales. Este método implica la detección de pequeñas descargas que se producen en huecos presentes dentro del dieléctrico. Los diferentes defectos dentro del aislamiento al ir progresando ocasionan un aumento en el nivel de descargas. Al seguir la tendencia de crecimiento de las descargas es posible planificar las paradas por mantenimiento y las reparaciones necesarias.

En los últimos años se han desarrollado equipos más avanzados que presentan especificaciones comerciales más específicas, según la necesidad de los usuarios. Estos equipos presentan tecnología digital y una amplia gama de opciones y usos por computadora, que facilitan la detección de fallas por descargas parciales para cualquier operador.

En sus estudios algunos autores [1], [3], realizaron el análisis de las descargas parciales en grandes máquinas rotantes, sin embargo, su análisis estuvo basado en la aplicación adecuada de la medición de diagnóstico y el monitoreo continuo on-line de descargas parciales, utilizando un acceso TCP/IP en una gran red.

Estos análisis sirvieron para la determinación de las descargas parciales y la supervisión de las mismas en las máquinas eléctricas puestas en funcionamiento. En tal sentido, la investigación realizada no ofrece clasificación de dichas fallas.

Estudios previos de las descargas parciales han permitido la detección de las mismas en las máquinas eléctricas, tal es el caso de otros autores [3] quienes desarrollaron una aplicación con redes neuronales probabilísticas en la detección de fallas incipientes en transformadores. Este estudio da solución al diagnóstico de gases contenidos en los aislantes de los equipos eléctricos. Este estudio permite adquirir las fallas presentes en transformadores sin involucrar el análisis particular de las mismas.

Otros métodos han permitido el estudio de las descargas parciales [1]-[4], [6] realizaron el diagnóstico en línea de motores de gran capacidad mediante la detección de descargas parciales, allí se plantea el análisis de las descargas parciales por la técnica de banda ultra ancha, logrando determinar algunos parámetros de las mismas.

Otro método empleado en el estudio de las descargas parciales, fue el de la transformada wavelet y la descomposición wavelet packet sobre señales de voltaje presentes en aisladores [7], [10], este método requirió de un gran número de cálculos previos y el estudio físico de las variables que intervienen. Tal investigación no involucró los patrones gráficos de descargas parciales.

Otros profesionales del área [6]-10] realizaron el análisis y muestreo de materiales aislantes para recubrimiento. Lograron mostrar que el escaneo microscópico de electrones arroja información de las cintas de corte del material aislante y la emisión infrarroja de la transformada de Fourier espectroscópica habilita la derivación de indicadores de material de oxidación que se desarrolla en la superficie. A diferencia de la investigación aquí planteada, donde se desarrolla el análisis espectral de los patrones tridimensionales de las descargas parciales y se realiza un procesamiento de las imágenes para lograr una reducción dimensional y finalmente coincidir en una red neuronal artificial.

Los patrones de descargas parciales presentan un gran número de datos estadísticos que permiten su caracterización [8]. \{11] haciendo uso de la herramienta de MATLAB para su análisis, logró determinar las variables involucradas con el patrón de descargas parciales.

\section{B.Tipos de descargas parciales}

Atendiendo a la definición de descargas parciales como un proceso de ruptura dieléctrica, en el cual el arco que se forma entre dos electrodos es de carácter parcial y transitorio, con un tiempo de duración muy corto y de un bajo contenido energético. Las descargas parciales se pueden caracterizar en tres tipos dependiendo de las propiedades del medio existente entre los electrodos: 


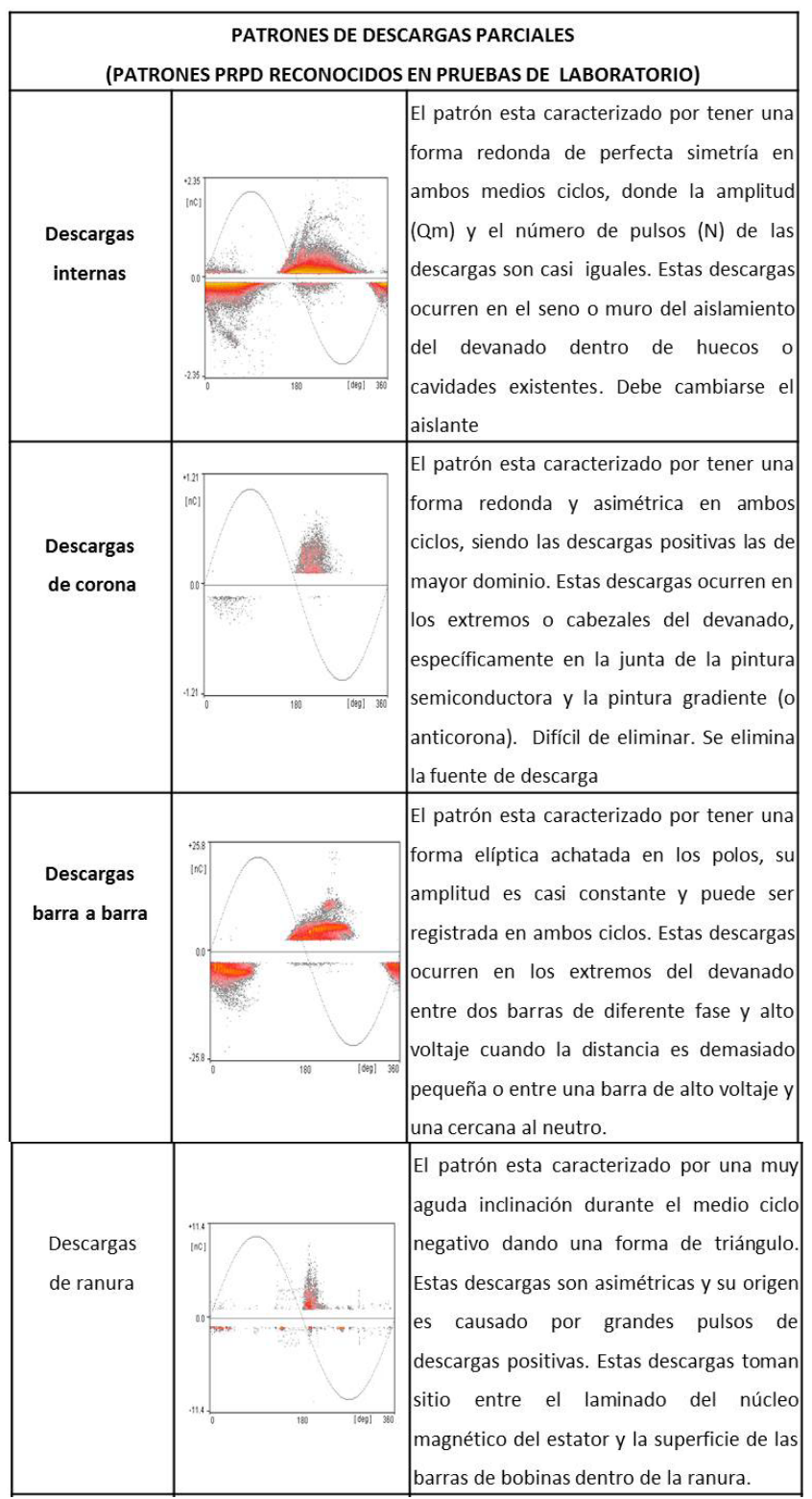

Fig. 1. Tipos de descargas parciales

C.Distribuciones Estadísticas

Como se ha descrito, el fenómeno de descargas parciales es un fenómeno estocástico y por tanto, para poder estudiarlo y analizarlo se debe obtener las distribuciones estadísticas de sus principales características en función del ángulo de fase del voltaje en que ocurren.

Por esta razón se trabaja con tres distribuciones estadísticas:

1) La distribución Hqmax $(\varphi)$ que muestra el valor máximo del pulso (pC) producido en cada ángulo de fase.

2) La distribución Hqn $(\varphi)$ que muestra el valor medio de la magnitud de los pulsos (pC) producidos en cada ángulo de fase.

3) La distribución Hn $(\varphi)$ que muestra el número de descargas producidas en cada ángulo de fase.

Las distribuciones son diferentes en el semiciclo positivo y en el semiciclo negativo del ciclo de la tensión. Por tanto, las tres distribuciones anteriormente definidas se dividirán en seis: H qmax $+(\varphi)$, Hqn $+(\varphi)$, Hn $+(\varphi)$ y $\operatorname{Hqmax}(\varphi), \operatorname{Hqn}(\varphi), \operatorname{Hn}(\varphi)$. 


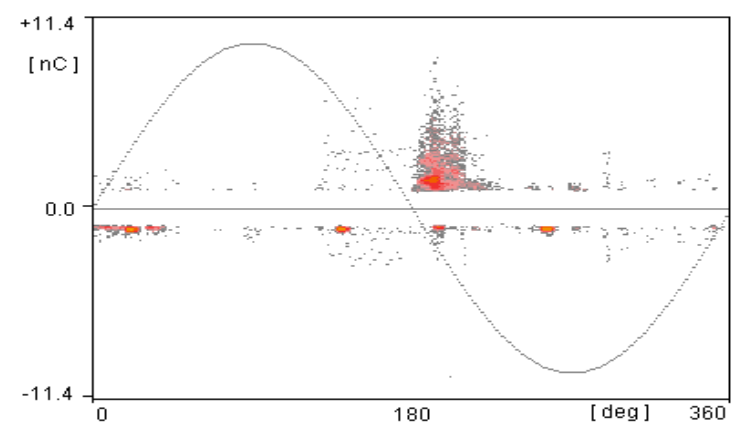

Fig. 2. Descarga tipo ranura

Tabla 1. Operadores estadísticos de la descarga tipo ranura

\begin{tabular}{|c|c|c|c|}
\hline & \multicolumn{3}{|c|}{ RANURA } \\
\hline $\begin{array}{l}\text { Estadís } \\
\text { tico }\end{array}$ & $\mathrm{Hqm}$ & Hqn & $\mathrm{Hn}$ \\
\hline $\mathrm{Sk}(\mathrm{U}+)$ & $-0,06$ & $-21,32$ & $-2,33$ \\
\hline Sk(U-) & 0,43 & $-22,45$ & 1,61 \\
\hline $\mathrm{Ku}(\mathrm{U}+)$ & $-1,58$ & $-23,32$ & 4,02 \\
\hline $\mathrm{Ku}(\mathrm{U}-)$ & $-1,32$ & $-24,45$ & 1,49 \\
\hline $\mathrm{Pe}(\mathrm{U}+)$ & 37 & $-25,32$ & 31 \\
\hline $\mathrm{Pe}(\mathrm{U}-)$ & 30 & $-26,45$ & 32 \\
\hline $\mathrm{Q}(\mathrm{U}+)$ & 15,13 & $-25,32$ & 35,33 \\
\hline $\mathrm{Q}(\mathrm{U}-)$ & 16,25 & $-26,45$ & $-36,46$ \\
\hline $\mathrm{cc}(\mathrm{U}+)$ & 17,13 & $-27,32$ & 37,33 \\
\hline $\mathrm{cc}(\mathrm{U}-)$ & 18,25 & $-28,45$ & $-38,46$ \\
\hline $\begin{aligned} & \operatorname{mcc}(\mathrm{U} \\
&+) \\
&\end{aligned}$ & 19,13 & $-29,32$ & 39,33 \\
\hline mcc(U- & 10,25 & $-20,45$ & $-30,46$ \\
\hline Est & 12,4837 & 2,7666 & 29,3490 \\
\hline
\end{tabular}

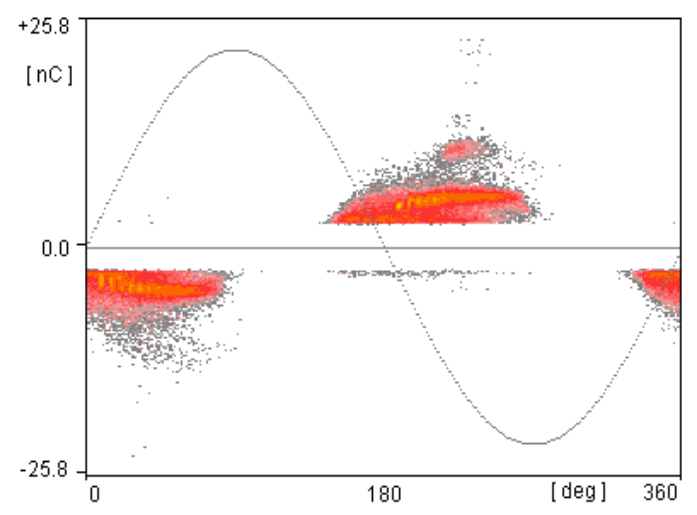

Fig. 3. Descarga parcial tipo barra-barra 
Tabla 2. Operadores estadísticos de la descarga tipo barra-barra

\begin{tabular}{|l|c|l|c|}
\hline & \multicolumn{3}{|c|}{ Barra-Barra } \\
\hline $\begin{array}{l}\text { Estadísti } \\
\text { co }\end{array}$ & Hqm & Hqn & Hn \\
\hline $\mathrm{Sk}(\mathrm{U}+)$ & 0,06 & $-21,32$ & 1,4 \\
\hline $\mathrm{Sk}(\mathrm{U}-)$ & 0,1 & $-22,45$ & 0,58 \\
\hline $\mathrm{Ku}(\mathrm{U}+)$ & $-1,15$ & $-23,32$ & 2,16 \\
\hline $\mathrm{Ku}(\mathrm{U}-)$ & $-1,11$ & $-24,45$ & 0,19 \\
\hline $\mathrm{Pe}(\mathrm{U}+)$ & 37 & $-25,32$ & 32 \\
\hline $\mathrm{Pe}(\mathrm{U}-)$ & 36 & $-26,45$ & 30 \\
\hline $\mathrm{Q}(\mathrm{U}+)$ & 15,13 & $-25,32$ & 35,33 \\
\hline $\mathrm{Q}(\mathrm{U}-)$ & 16,25 & $-26,45$ & $-36,46$ \\
\hline $\mathrm{cc}(\mathrm{U}+)$ & 17,13 & $-27,32$ & 37,33 \\
\hline $\mathrm{cc}(\mathrm{U}-)$ & 18,25 & $-28,45$ & $-38,46$ \\
\hline $\mathrm{mcc}(\mathrm{U}+)$ & 19,13 & $-29,32$ & 39,33 \\
\hline $\mathrm{mcc}(\mathrm{U}-)$ & 10,25 & $-20,45$ & $-30,46$ \\
\hline $\begin{array}{l}\text { Desv- } \\
\text { Est }\end{array}$ & 13,255769 & 2,7666417 & 29,25211 \\
\hline
\end{tabular}

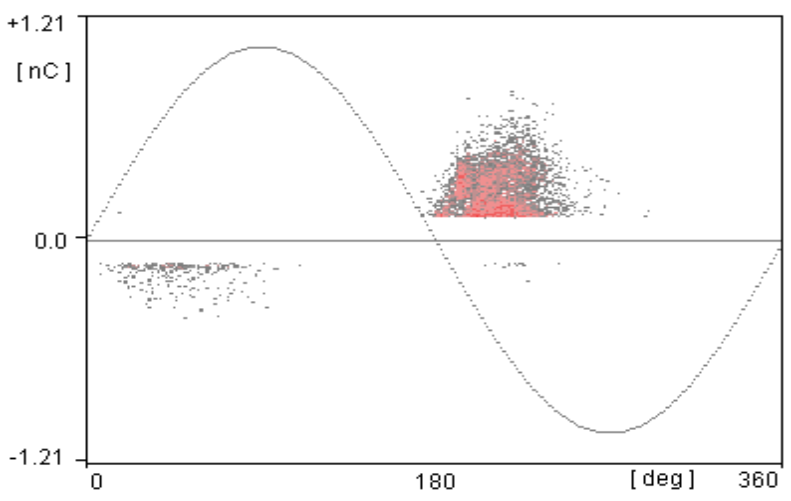

Fig. 4. Descarga tipo corona

Tabla 3. Operadores estadísticos de la descarga tipo corona

\begin{tabular}{|l|c|c|c|}
\hline & \multicolumn{3}{|c|}{ Interna } \\
\hline $\begin{array}{l}\text { Estadísti } \\
\text { co }\end{array}$ & $\mathrm{Hqm}$ & $\mathrm{Hqn}$ & $\mathrm{Hn}$ \\
\hline $\mathrm{Sk}(\mathrm{U}+)$ & 0,4 & $-21,32$ & 2,55 \\
\hline $\mathrm{Sk}(\mathrm{U}-)$ & 0,45 & $-22,45$ & 1,78 \\
\hline $\mathrm{Ku}(\mathrm{U}+)$ & $-1,15$ & $-23,32$ & 5,92 \\
\hline $\mathrm{Ku}(\mathrm{U}-)$ & $-0,84$ & $-24,45$ & 5,88 \\
\hline $\mathrm{Pe}(\mathrm{U}+)$ & 42 & $-25,32$ & 13 \\
\hline $\mathrm{Pe}(\mathrm{U}-)$ & 33 & $-26,45$ & 17 \\
\hline $\mathrm{Q}(\mathrm{U}+)$ & 15,13 & $-25,32$ & 35,33 \\
\hline $\mathrm{Q}(\mathrm{U}-)$ & 16,25 & $-26,45$ & $-36,46$ \\
\hline $\mathrm{cc}(\mathrm{U}+)$ & 17,13 & $-27,32$ & 37,33 \\
\hline $\mathrm{cc}(\mathrm{U}-)$ & 18,25 & $-28,45$ & $-38,46$ \\
\hline $\mathrm{mcc}(\mathrm{U}+)$ & 19,13 & $-29,32$ & 39,33 \\
\hline $\mathrm{mcc}(\mathrm{U}-)$ & 10,25 & $-20,45$ & $-30,46$ \\
\hline Desv-Est & 13,610008 & 2,7666417 & 27,353579 \\
\hline
\end{tabular}




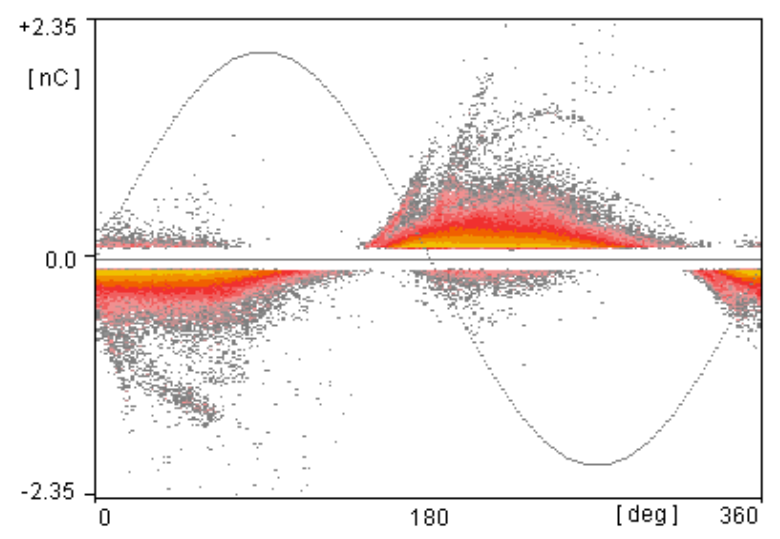

Fig. 5. Descarga tipo interna

Tabla 4. Operadores estadísticos de la descarga tipo interna

\begin{tabular}{|l|c|c|c|}
\hline & \multicolumn{3}{|c}{ Interna } \\
\hline $\begin{array}{l}\text { Estadísti } \\
\text { co }\end{array}$ & Hqm & Hqn & Hn \\
\hline $\mathrm{Sk}(\mathrm{U}+)$ & 0,4 & $-21,32$ & 2,55 \\
\hline $\mathrm{Sk}(\mathrm{U}-)$ & 0,45 & $-22,45$ & 1,78 \\
\hline $\mathrm{Ku}(\mathrm{U}+)$ & $-1,15$ & $-23,32$ & 5,92 \\
\hline $\mathrm{Ku}(\mathrm{U}-)$ & $-0,84$ & $-24,45$ & 5,88 \\
\hline $\mathrm{Pe}(\mathrm{U}+)$ & 42 & $-25,32$ & 13 \\
\hline $\mathrm{Pe}(\mathrm{U}-)$ & 33 & $-26,45$ & 17 \\
\hline $\mathrm{Q}(\mathrm{U}+)$ & 15,13 & $-25,32$ & 35,33 \\
\hline $\mathrm{Q}(\mathrm{U}-)$ & 16,25 & $-26,45$ & $-36,46$ \\
\hline $\mathrm{cc}(\mathrm{U}+)$ & 17,13 & $-27,32$ & 37,33 \\
\hline $\mathrm{cc}(\mathrm{U}-)$ & 18,25 & $-28,45$ & $-38,46$ \\
\hline $\mathrm{mcc}(\mathrm{U}+)$ & 19,13 & $-29,32$ & 39,33 \\
\hline $\mathrm{mcc}(\mathrm{U}-)$ & 10,25 & $-20,45$ & $-30,46$ \\
\hline Desv-Est & 13,610008 & 2,7666417 & 27,353579 \\
\hline
\end{tabular}

La extracción de los operadores estadísticos permitió evaluar la similitud intrínseca de estos patrones. Se observó que aun siendo distintos estos patrones; sus diferencias no son relevantes para garantizar un análisis de clasificación de los mismos usando técnicas neuronales. Lo cual indica que estos operadores no pueden ser empleados para diferenciarlas una de la otra y por ende, no pueden ser empleados para la clasificación.

En virtud de que los datos estadísticos no eran suficientes para garantizar una clasificación, se optó por hacer un clúster a partir de las imágenes propiamente. De esta manera resultaría evidente la discriminación entre ellas.

Para el análisis de las imágenes fue necesario realizar un filtrado previo de las mismas. Con la intención de simplificar los datos, removiendo toda aquella información que no es de interés. La simplificación controla la cantidad y naturaleza de la información que es preservada. Además los datos simplificados deben contener áreas fáciles de segmentar. En este caso particular, las imágenes se han simplificado eliminando el excedente de los bordes de las imágenes que no contiene información de interés para el procesado, adicional a ello se han extraído los planos RGB para así poder convertirla a binaria para poder emplearlas en MATLAB.

Para el análisis de las imágenes de descargas parciales se realizaron tres procedimientos distintos, los cuales 
consistieron en tres métodos de procesamiento de los datos.

Método 1: una vez procesada la imagen, los datos se cargan en un arreglo bidimensional, en el cual se pueden observar los cuatro tipos de descargas parciales en un mismo plano, agrupadas como un conjunto de puntos y rayas, según el tipo de descarga. Este nuevo patrón es cargado al mapa auto organizativo

En vista que las descargas parciales proporcionan gran número de datos, se utilizó un Mapa Auto Organizativo (SOM) para realizar una reducción dimensional de la misma y a la vez una clasificación de las características.

Una vez realizada la arquitectura del SOM, se procedió a evaluar los resultados del mismo. El mapa auto organizativo permitió caracterizar las descargas y separarlas según el tipo de datos suministrados.

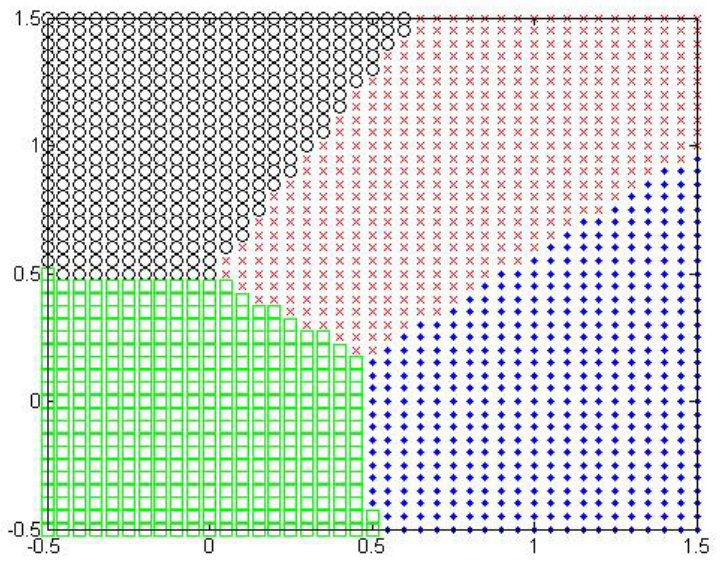

Fig. 6. Mapa del SOM

Método 2: En este método se filtró la imagen, de la misma manera que en el método 1, pero a diferencia del primero, se cargaron esos datos en el SOM para luego extraer la matriz de pesos del SOM. A diferencia del método 1, éste método toma los pesos del SOM para luego procesarlos. Mientras que el método 1, toma el arreglo con las cuatro imágenes. Sin embargo este método arrojó resultados aceptables pero con un margen de error mayor que el primero.

Método 3: Este método filtra la imagen de la misma forma que los métodos anteriores, pero extrae los datos para ser procesados en una red de tipo backpropagation. Este método resultó ser el más confiable con el menor porcentaje de error.

\section{IV.CONCLUSIONES}

El análisis de las descargas parciales a partir de técnicas neuronales permitió establecer una similitud entre los operadores estadísticos de las distintas descargas. Lo cual resultó de interés para su posterior análisis.

El estudio de clasificación con técnicas neuronales fue posible por tres métodos diferentes. Logrando diferenciar una de la otra a partir de sus matrices de pesos y de sus características gráficas.

La clasificación de los patrones de descargas parciales se logró a partir de la imagen de cada descarga, logrando establecer un algoritmo neuronal apropiado para el procesamiento de imágenes bidimensionales.

\section{REFERENCIAS}

[1]Grimón, Y. (2012) Algoritmo de clasificación de imágenes usando redes neuronales.

[2]Cadena, J. Cadena J. (2008) Aplicación de redes neuronales probabilísticas en la detección de fallas incipientes en transformadores. Universidad Tecnológica de Pereira

[3]Carvajal, A. García, V. Asiaín, T. (2008) Diagnóstico en línea de motores de gran capacidad mediante la detección de descargas parciales utilizando técnicas de banda ultra ancha. Revista información tecnológica vol. 19 $\mathrm{N}^{\circ} 1$ enero 2008. Centro e información tecnológica, La Serena, Chile. 
[4]Krivda, A., blackmore, P. y Birtwhistle, D. "Materials in overhead distribution networks. IEEETansactions on dielectrics and electrical insulation". Vol. 6. N 51999

[5]Magrashi, A. (1997) Pattern recognition of partial discharges using matlab tools. Sultan Qaboos University, Oman.

[6]Suárez, F. "Proceso Neuronal para Análisis de Descargas Parciales en la empresa CORPOELEC, de Ciudad Guayana, Venezuela”. Rev. Hallazgos 21. Vol. 3, Num. 02. Pp 169-180. Junio 2018

[7]Suárez, F., Sucre, P., Almeida, G., "Inteligencia artificial en el proceso de obtención de alumina”, Rev. Universidad, Ciencia y Tecnología. Vol. 22, Num. 88. Pp. 48-56. Julio 2019.

[8]Gómez, M. (2007) “Ejemplo de aplicación de una red de retropropagación” [En línea] Disponible en www. iiia. CISC. Es

[9]Nooraii, A. (2003) "Applying Multivariable Control Technology At Alcoa World Alumina" (Paper presented at the Honeywell User's Group Annual Meeting, Brisbane, Australia.

[10]Rumelhard, D., Hinton, G \& Willians, R. (1986)"Learning representations by backpropagation errors". Neture.

[11]Bryson, A. HO, Y. (1969) "Applied optimal control" Blaisted. New York.

[12]Mason, D. (1998) "Process Control Technology Saving Energy" Australian Energy News, 10. 